\title{
Molecular Dynamics Simulations of Crater Formation Induced by Laser Ablation on the Surface of $\alpha-F e$ Substrate
}

\author{
Jenn-Kun Kuo ${ }^{1}$, Pei-Hsing Huang ${ }^{2, a}$, Shih-Kai Chien ${ }^{3}$, Kuang-Yao Huang ${ }^{3}$ and Kun-Tso Chen ${ }^{3}$ \\ ${ }^{1}$ National University of Tainan, Department of Greenergy, 70005 Tainan, Taiwan \\ ${ }^{2}$ National Pingtung University of Science and Technology, Department of Mechanical Engineering, 912 Pingtung, \\ Taiwan \\ 3ITRI SOUTH Laser and Manufacturing Technology Center (LAMC), Laser Manufacturing System Department, , \\ 73445 Tainan, Taiwan
}

\begin{abstract}
In this study, we employed molecular dynamics (MD) simulations to investigate ablation induced by the application of pulsed laser irradiation to a thin $\alpha-\mathrm{Fe}$ substrate. We observed several mechanisms underlying the removal of material, including ultrafast melting, cluster ejection, and thermal vaporization. We also examined the effects of laser fluence on the resulting surface morphology as well as the amount of material ablated and deposited around the craters
\end{abstract}

\section{Introduction}

Laser cutting has been shown to outperform conventional oxyacetylene cutting with regard to speed and quality while enabling non-contact processing. This technology has been adopted in numerous industrial applications, as a good compromise between efficiency and accuracy. The removal and cutting of materials using laser ablation involves two primary mechanisms: vaporization and melt ejection [1-4]. Achieving a high-quality laser cutting surface requires a basic understanding of the underlying processes; however, observations of laser cutting are limited to extremely short time periods. Furthermore, this process involves complex interactions among a variety of phases. In this study, we used numerical simulations to directly observe the transient phenomena associated with laser cutting and ablation [5-8].

Laser-induced structural changes occur on extremely short timescales, falling somewhere between the natural oscillation cycles of an atom or molecule and several picoseconds. These changes are induced by a complex combination of processes, including the transmission of light energy, carrier dynamics, heating, and mechanical processes. As a result, the fundamental mechanisms underlying laser ablation have yet to be fully elucidated, which has hindered the further development of this technology for manufacturing [9-12]. In this study, we adopted molecular dynamics (MD) at the microscopic scale to explore structural metamorphoses induced by light, heat, and mechanical processes. We used simulations to conduct a qualitative study on the interactions between lasers and metal targets followed by experimental verification of our results. Our overall objective was to enhance the precision laser cutting and thereby improve the quality of laser cut surfaces.

\footnotetext{
${ }^{\mathrm{a}}$ Corresponding author : phh@mail.npust.edu.tw
} 


\section{Numerical Methods}

MD simulation was used to investigate the ablation of a thin $\alpha$-Fe substrate. Laser energy applied to the substrate decayed exponentially in the direction of incident light in accordance with the BeerLambert law. The energy of the photons was transformed into kinetic energy (in the irradiated atoms) within a characteristic time $\left(\tau_{\text {eq }}\right)$ [8]. Time $\tau_{\text {eq }}$ corresponds to the relaxation time between electrons and the lattice, which typically takes between hundreds of femtoseconds to several tens of picoseconds for metals, depending on the properties of the target $[1,10,11]$. Here, we adopted a typical relaxation time of $\tau_{\mathrm{eq}} \sim 10 \mathrm{ps}$. The laser energy deposited to depth $\mathrm{z}$ within the substrate is expressed using the equation of $\mathrm{S}(\mathrm{z})$ as follows:

$$
S(z)=I_{0}(1-R) D_{p}^{-1} e^{-z / D_{p}}
$$

where $I_{0}$ is the intensity of the laser radiation, R denotes the reflectivity of the substrate, and $D_{p}$ is the optical penetration depth. We accounted for effects of electron thermal diffusion as an increase in the effective depth of penetration by the laser, wherein depth was estimated using the electron thermal diffusion length [2]. The physical parameters used in the simulation are listed in Table 1.

Table 1. Physical parameters of $\alpha$-Fe used in the simulation.

\begin{tabular}{|c|c|c|c|c|}
\hline$a_{0}(\mathrm{~nm})$ & $\mathrm{R}$ & $\mathrm{D}_{\mathrm{p}}(\mathrm{nm})$ & $\Delta t(\mathrm{fs})$ & $r_{\mathrm{e}}(\mathrm{nm})$ \\
\hline 0.286595 & $65 \%$ & 18.5 & 5 & 2.481987 \\
\hline
\end{tabular}

The EAM potential energy model developed by Wadley et al. [13] was used in the MD simulations to describe interactions between atoms. The EAM model is in good agreement with the basic material properties (e.g., lattice constant, elastic constant, bulk modulus, sublimation energy, and heat of solution) of the substrate [14]. The total energy Etot in this system of atoms is defined as follows [13]:

$$
\begin{gathered}
E_{\text {total }}=\sum_{i} F_{i}\left(\rho_{h, i}\right)+\frac{1}{2} \sum_{i, j(i \neq j)} \phi_{i j}\left(r_{i j}\right) \\
\rho_{h, i}=\sum_{j \neq i} f\left(r_{i j}\right)
\end{gathered}
$$

where the summation includes the total number of atoms in the system. Embedded energy, $F_{i}$, is a function of the host electron density $\rho_{h, i}$ induced at the site of atom $i$ by all other atoms in the system. Pair potential, $\phi_{i j}$, is a function of the distance $r_{i j}$ between atom $i$ and atom $j$. re is the equilibrium distance between the nearest neighbor atoms [14]. The evolution of atomic dynamic trajectories is governed by Hamilton's classical equation based on Newton's second law of motion. The force acting on atom i can be calculated according to the gradient of potential energy as follows:

$$
\begin{aligned}
\vec{F}_{i} & =-\nabla_{\vec{r}_{i}} E_{\text {tot }} \\
& =-\sum_{j, j \neq i}\left[\frac{\partial F_{i}\left(\rho_{i}\right)}{\partial \rho_{i}} \frac{\partial f_{j}\left(r_{i j}\right)}{\partial r_{i j}}+\frac{\partial F_{j}\left(\rho_{j}\right)}{\partial \rho_{j}} \frac{\partial f_{i}\left(r_{i j}\right)}{\partial r_{i j}}+\frac{\partial \phi_{i j}\left(r_{i j}\right)}{\partial r_{i j}}\right] \frac{\vec{r}_{i}-\vec{r}_{j}}{r_{i j}}
\end{aligned}
$$

The potential energy parameters used in the EAM model are detailed in Ref. [13]. Before initiating the simulation, the atoms in the system were randomly assigned atomic velocities appropriate to room temperature $(300 \mathrm{~K})$ for a given simulation, in accordance with Maxwell-Boltzmann distribution [15]. Periodic boundary conditions were applied to the atoms in the $\mathrm{x}$ direction of the computational domain. Free boundary conditions were assumed in the $y$ and $\mathrm{z}$ directions, under which Fe atoms were permitted to travel straight along the two directions without any restrictions. Ablation was simulated at a pulse duration in which the electron-phonon relaxation time was 10.0 ps where the photonic wavelength of $\lambda=800 \mathrm{~nm}$. The optical parameters of the material used in the calculations at this 
wavelength were as follows: reflectivity $\mathrm{R}=65 \%$ and optical penetration depth $=18.5 \mathrm{~nm}$ [2]. The laser pulse was focused at the center of the simulation system with an even spatial distribution of intensity along the $x-y$ plane. The ablation process was performed under vacuum. Gear's fifth-order predictor-corrector algorithm [15] was used to estimate the positions, velocities, and accelerations of the atoms, as well as the interaction forces between atoms. This algorithm features excellent numerical stability and provides a satisfactory compromise between time consumption and precision [16].

\section{Results and Discussion}

Before initiating the MD simulations, we adopted the embedded atom method (EAM) to describe the interactions among metallic atoms. To ensure accuracy, we performed a heating and melting point experiment on $10 \times 10 \times 10 a_{0}$ Fe-BCC crystal material ( $a_{0}$ represents the lattice constant of $\alpha-\mathrm{Fe}$ ). The $\lambda$-function [15] was used to identify the degree of order in the periodic arrangement of the lattice. A $\lambda$ function value approaching 1 indicates that the substrate is in solid state, a lower $\lambda$-function value refers to a less ordered state, and a $\lambda$-function approaching 0 means that the substrate is in a completely molten state. The simulation results in Fig. 1 clearly illustrate the changes in the $\lambda$ function and crystal structure as the heating time was increased. $\lambda$-function rapidly decreased with an increase in temperature. When the temperature rose to between $1,200 \mathrm{~K}$ and $1,400 \mathrm{~K}$, the $\lambda$-function tended to remain constant. This period falls within the stage of allotropic transformation in the $\alpha-\mathrm{Fe}$ structure. At a temperature of $1,850 \mathrm{~K}$, the crystal began melting, such that the $\lambda$-function value fell to 0 . At room temperature, the atomic polymerization energy of the crystal was $\sim 4.28 \mathrm{eV} / \mathrm{atom}$, which is consistent with calculation results obtained via quantum mechanics [15]. These results prove the accuracy of the EAM and MD simulations used in this study.

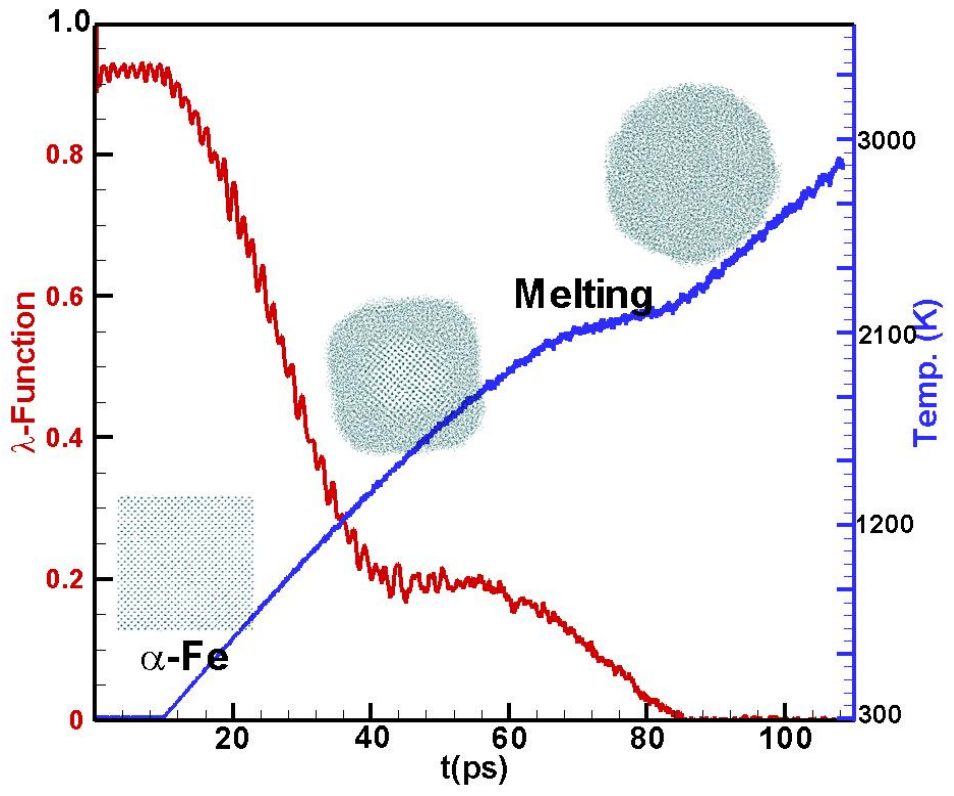

Figure 1. Time evolution of temperature and relationship between $\lambda$-function and crystal structure in molecular dynamics simulations of $\alpha$-Fe undergoing heating.

Figure 2. illustrates the ablation state resulting from the application of a laser spot with diameter of $4.0 \mathrm{~nm}$ and laser energy of $600 \mathrm{~mJ} / \mathrm{cm}^{2}$ to a $50 \times 50 \times 50 a_{0}(14.3 \times 14.3 \times 4.29 \mathrm{~nm}) \mathrm{Fe}-\mathrm{BCC}$ crystal. The colors of the atoms in the figure indicate their potential energy values. Under laser irradiation, the rapid increase in temperature led to ultrafast melting in the substrate at the location of the laser spot. At time $t=17 \mathrm{ps}$, phase eruption and cluster ejection were observed in many atoms within the surface 
layer. Once a certain number of the melted atoms had been ablated, they did not undergo complete desorption, and neither did they lift-off from the substrate surface. Thus, this process led the formation of a crater deposit.

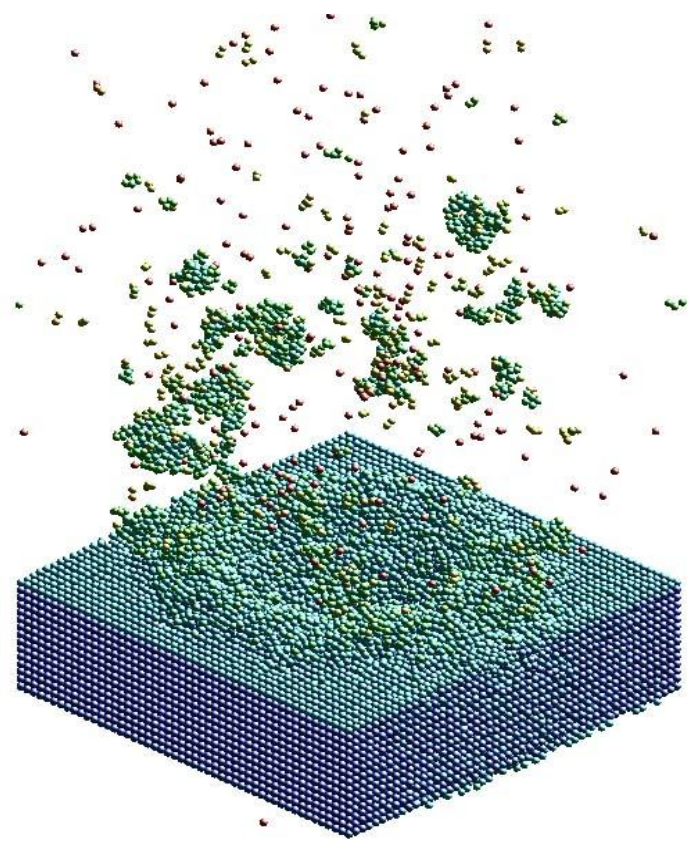

Figure 2. Ablation state resulting from the radiation of laser fluence of $600 \mathrm{~mJ} / \mathrm{cm}^{2}$ to Fe-BCC crystal.

Figure 3. presents changes in average temperature in the substrate when heated using a laser of various energies. The lattice heating process relies on the intensity of electron-phonon scattering; therefore, we assumed that the electron-phonon relaxation time was $\tau_{\text {eq }} \sim 10 \mathrm{ps}$ in this study. As shown in Fig. 3, the average temperature of the substrate increased rapidly from the onset of heating. After being heated using laser energies of $300 \mathrm{~mJ} / \mathrm{cm}^{2}, 600 \mathrm{~mJ} / \mathrm{cm}^{2}$, and $900 \mathrm{~mJ} / \mathrm{cm}^{2}$, the average temperature in the substrate respectively peaked at $2,385 \mathrm{~K}, 3,064 \mathrm{~K}$, and $3,962 \mathrm{~K}$ before gradually declining.

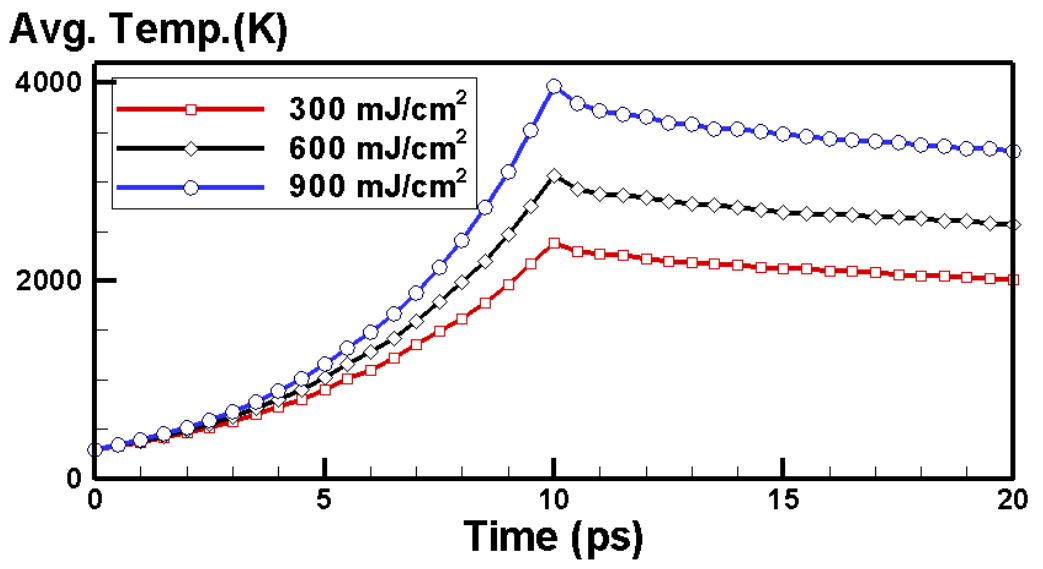

Figure 3. Time-evolution of average temperature in $\alpha$-Fe substrate.

During the laser heating process, the surface and the interior of the target underwent rapid heating and swelling, which caused the eruption of melted as well as semi-melted material from the top and 
bottom surfaces. Laser energy of $900 \mathrm{~mJ} / \mathrm{cm}^{2}$ was sufficient to penetrate the substrate and form micropores, whereas laser energy of $300 \mathrm{~mJ} / \mathrm{cm}^{2}$ was insufficient to penetrate the substrate and merely formed a small pit. Melted material that did not undergo complete desorption or lift-off from the substrate surface (i.e.. residual slag or residue) created a crater-like structure, which severely reduced the quality of the surface (Fig. 4).

(a)

(b)

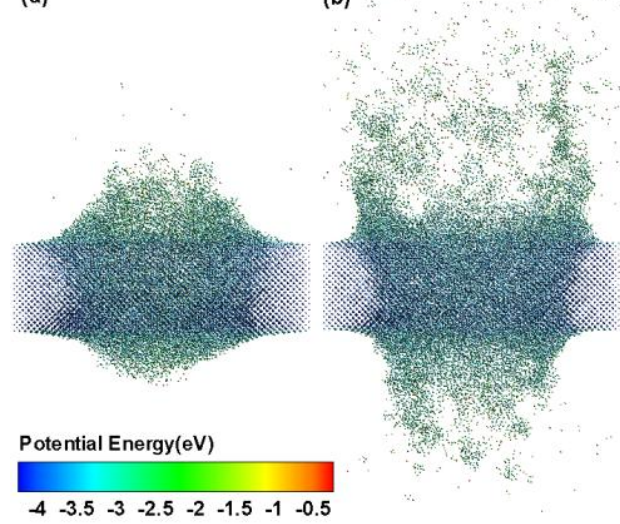

(b) (c)

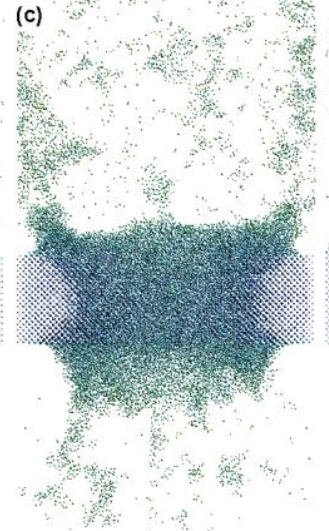

(d)

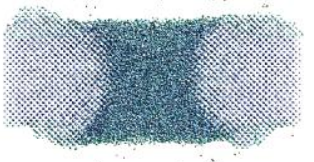

Figure 4. (a)-(d) Transient states of $\alpha$-Fe crystal irradiated at a laser energy of $900 \mathrm{~mJ} / \mathrm{cm}^{2}$ for $t=10 \mathrm{ps}, 15 \mathrm{ps}, 20$ ps, and 50 ps.

As shown in Fig. 5(a), the application of a laser with energy of $\mathrm{F} \leq 600 \mathrm{~mJ} / \mathrm{cm}^{2}$ resulted in a larger number of atoms being ablated from the top surface $\left(N_{a}\right)$. At this energy level, deformations also formed on the bottom surface, due to stress induced by the thermal shock wave. Nonetheless, the fact that the laser did not penetrate the target meant that almost no atoms were ablated from the bottom surface, as shown in Fig. 5(b). In contrast, higher energy levels enabled the laser to penetrate the substrate, which resulted in the formation of micropores (Fig. 4). In these cases, the number of atoms passing through the bottom surface exceeded the number of atoms ablated from the top surface. Nonetheless, a portion of the metal slag lacked the ejection or lift-off power required to overcome the attraction of the metal bonds. These atoms did not undergo complete desorption or lift-off from the target surface in the final stage, such that $N_{a}$ ultimately decreased. These atoms eventually accumulated on the top and bottom surfaces of the target to form uneven craters and bumps.
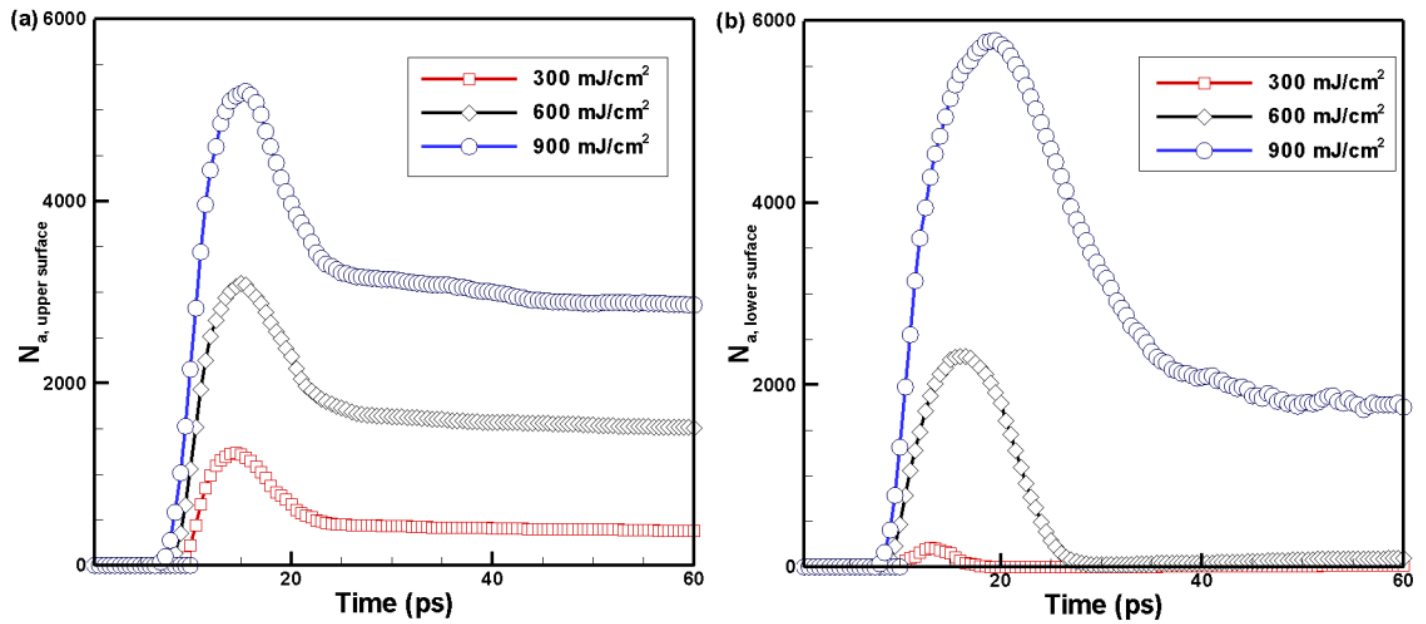

Figure 5. (a)-(d) Number of melted atoms that underwent desorption from (a) top surface and (b) bottom surface of substrate during laser heating. 


\section{SUMMARY}

In this study, EAM molecular dynamics was used to simulate the laser ablation of $\alpha$-Fe surfaces in order to elucidate the mechanisms underlying the formation of craters. Our results could be used to guide the design of process parameters with the objective of improving the quality of laser-cutting surfaces.

\section{Acknowledgements}

The authors gratefully acknowledge the support provided for this research by the ITRI SOUTH Laser and Manufacturing Technology Center under grant C106-078 and by the Ministry of Science and Technology, R.O.C. under grants MOST 106-2221-E-020-014 and MOST 106-2622-E-020-004-CC3. We would also like to thank the National Center for High-performance Computing (NCHC) of National Applied Research Laboratories (NARLabs) of Taiwan for providing computational and storage resources.

\section{References}

1. P.-H. Huang, H.Y. Lai, Phys. Rev. B 77, 125408, 12 pages (2008)

2. N.N. Nedialkov, S.E. Imamova, P.A. Atanasov, G. Heusel, D. Breitling, A. Ruf, H. Hugel, F. Dausinger, P. Berger, Thin Solid Films 453-454, 496-500 (2004)

3. P.-H. Huang, H.Y. Lai, J. Appl. Phys. 108, 123504, 10 pages (2010)

4. H.Y. Lai, P.-H. Huang, Comput. Mater. Sci. 41, 498-507 (2008)

5. S.E. Imamova, P.A. Atanasov, N.N. Nedialkov, F. Dausinger, P. Berger, Nucl. Instrum. Methods Phys. Res. Sect. B-Beam Interact. Mater. Atoms 227, 490-498 (2005)

6. P.-H. Huang, H.Y. Lai, J. Chin. Soc. Mech. Eng. 31, 477-483 (2010)

7. H.Y. Lai, P.-H. Huang, Appl. Surf. Sci. 254, 3067-3073 (2008)

8. N.N. Nedialkov, P.A. Atanasov, Appl. Surf. Sci. 252, 4411-4415 (2006)

9. H.Y. Lai, P.-H. Huang, J. Chin. Soc. Mech. Eng. 28, 577-583 (2007)

10. N. Nedialkov, P. Atanasov, S. Imamova, J. Phys. D 36, 638(2004).

11. P.-H. Huang, H.Y. Lai, Nanotechnology, 19, 255701, 11 pages (2008)

12. H.Y. Lai, P.-H. Huang, T.H. Fang, Appl. Phys. A-Mater. Sci. Process. 86, 497-503 (2007)

13. H.N.G. Wadley, X. Zhou, R.A. Johnson, M. Neurock, Prog. Mater. Sci. 46, 329 (2001)

14. P.-H. Huang, J.K. Kuo, Appl. Phys. A-Mater. Sci. Process. 103, 1083-1092 (2011)

15. J.M. Haile, Molecular dynamics simulation: elementary methods, Wiley-Interscience, New York. (1997)

16. P.-H. Huang, Compos. Sci. Technol. 72, 599-607 (2012) 